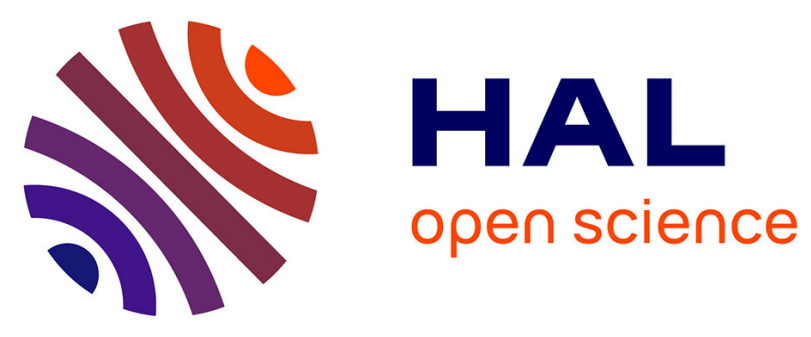

\title{
Multivariable Generalized Bouc-Wen modeling, identification and feedforward control and its application to multi-DoF piezoelectric actuators.
}

Didace Habineza, Micky Rakotondrabe, Yann Gorrec

\section{- To cite this version:}

Didace Habineza, Micky Rakotondrabe, Yann Gorrec. Multivariable Generalized Bouc-Wen modeling, identification and feedforward control and its application to multi-DoF piezoelectric actuators.. The 19th World Congress of the International Federation of Automatic Control. IFAC'14, 2014, Cape Town, South Africa. pp.10952-10958. hal-01324728

\section{HAL Id: hal-01324728 \\ https://hal.science/hal-01324728}

Submitted on 1 Jun 2016

HAL is a multi-disciplinary open access archive for the deposit and dissemination of scientific research documents, whether they are published or not. The documents may come from teaching and research institutions in France or abroad, or from public or private research centers.
L'archive ouverte pluridisciplinaire HAL, est destinée au dépôt et à la diffusion de documents scientifiques de niveau recherche, publiés ou non, émanant des établissements d'enseignement et de recherche français ou étrangers, des laboratoires publics ou privés. 


\title{
Multivariable Generalized Bouc-Wen modeling, identification and feedforward control and its application to multi-DoF piezoelectric actuators
}

\author{
Didace Habineza, Micky Rakotondrabe and Yann Le Gorrec \\ Automatic Control and Micro-Mechatronic Systems depart. (AS2M), \\ FEMTO-ST Institute, CNRS - University of Franche-Comté at \\ Besançon (UFC) - ENSMM - UTBM, Besançon France. \\ e-mails:\{didace.habineza; mrakoton; legorrec\}@femto-st.fr
}

\begin{abstract}
In the literature, the generalized Bouc-Wen model can track precisely asymmetric hysteresis nonlinearity. In this paper, we propose to extend this generalized model to multivariable hysteresis model that can track the nonlinearities in multi-degrees of freedom (multi-DoF) hysteretic actuated systems. In particular, these systems are typified by strong hysteresis couplings. Then, a method of identification of the multivariable hysteresis model is proposed. Finally, based on the inverse multiplicative structure, we propose a multivariable feedforward compensator of the nonlinearity. The proposed approach has been applied to a multiDoF piezoelectric tube (piezotube) used in scanning probe microscopy and the experimental verification demonstrated its validity in terms of model precision and compensation efficiency.
\end{abstract}

Keywords: Feedforward Control, Multi-Degrees of Freedom Systems, Asymmetric Hysteresis, Couplings, Generalized Bouc-Wen Model.

\section{INTRODUCTION}

Hysteresis is very known to be one of the piezoelectric actuators behaviours that compromise the positioning accuracy. Hysteresis control has been largely studied either by using feedback or feedforward control techniques.

In some applications such as precise positioning and micromanipulation, the use of feedback control techniques to remove hysteresis are limited by the lack of convenient sensors having the required performances and convenient dimensions. In fact, embeddable sensors such as strain gauges do not possess the required performances while performant sensors such as optical sensors are very bulky [6][8]. For this reason, feedforward (open-loop) techniques have been adopted.

Two approaches have used feedforward control hysteresis in piezoelectric systems. The first approach consists in modeling the hysteresis and then apply a model inversion, in order to establish a linear relation between the input and output displacement [11][8]. This approach is called voltage control. The second approach is the charge control where, instead of inverting the hysteresis model, the input to the system is no longer the voltage, but the electric charge provided by a designed electronic circuit [10]. This latter approach benefits from the linearity between the input charge and the output displacement of the piezoelectric actuator.

* This work is supported by the national ANR-JCJC C-MUMSproject (National young investigator project ANR- 12-JS03007.01: Control of Multivariable Piezoelectric Microsystems with Minimization of Sensors).
Hysteresis modeling for smart materials actuators has been widely studied [12][22]. For piezoelectric actuators, the Preisach, the Prandtl-Ishlinskii and the Bouc-Wen models are the most used if the further aim is to calculate a feedforward controller. The two first are based on the superposition of many elementary hysteresis operators (hysterons) [11][13][19][20], while the third lies on a set of two equations made of a number of parameters controlling the shape and the scale of hysteresis [6] [4].

In the classical models of the above techniques (Preisach, Prandtl-Ishlinskii, Bouc-Wen), the hysteresis modelled are symmetrical. However, many hysteresis behaviour encountered in piezoelectric actuators like piezo-stack actuators [14], piezoelectric multimorph cantilevers [17], piezotube scanners, etc, are non-symmetrical. To model this class of hysteresis, additional models have been introduced (Dahl [18]), and some of the existing models have been extended or modified: Extended Coleman-Hodgdon [9], Modified Preisach [7], Modified Prandtl-Ishlinskii [2] and Generalized Bouc-Wen [1].

The asymmetric hysteresis models cited above are not easily extensible to multi-DoF systems. In addition, in the case of multiple degrees of freedom (multi-DOF), the number of parameters to be identified can be very high leading to a very complex calculation and implementation of the compensator. In fact, the Preisach and the PrandtlIshlinskii techniques present these disadvantages relative to the Generalized Bouc-Wen technique. Indeed, the two formers being based on the superposition of many elementary hysteresis, extending them to multivariable implies a substantial increase of the parameters. In the Generalized 
Bouc-Wen technique, the number of parameters will still be conveniently limited if extended in multivariable offering a simplicity of model identification and of compensator calculation and implementation.

In the literature, the study of multivariable hysteresis is not yet well addressed. [15][16][18] focused on the modeling of multivariable hysteresis which are symmetrical. In [9], the compensation of hysteresis in a multi-DoF piezoelectric actuator has been considered. However, only the direct transfers have been controlled and the couplings were not considered. The works come back to the feedforward control of several monovariable hysteresis. In [14][17], couplings were taken into account but their suppression were performed by combining the feedforward and the feedback schemes. In this paper we propose the modeling, the parameters identification and the feedforward control of asymmetrical hysteresis in multi-DoF piezoelectric actuators. The proposed hysteresis model and compensator are multivariable because they account both the direct hysteresis and the coupling hysteresis. In order to benefit from the model simplicity of the Generalized Bouc-Wen model [1], we propose in this paper to extend such monovariable hysteresis model into a multivariable hysteresis model. Then, we propose a procedure of identification of its parameters. Afterwards, a new compensator of the multivariable hysteresis is proposed. The compensation technique is based on the inverse multiplicative structure and on the identified model. The advantage of this proposed compensator is: as soon as the model is identified, the compensator is yielded without additional calculation thanks to the structure. Finally, experiments on a piezotube scanner validate the efficiency of the proposed modeling, identification and compensation approach.

The remainder of this paper is as follows. Section- 2 reminds the existing monovariable generalized Bouc-Wen asymmetrical hysteresis model. In section-3, its extension into multivariable hysteresis model is presented. The proposed procedure of parameters identification is also detailed in the same section. Section- 4 is devoted to the compensator calculation for the multivariable asymmetrical hysteresis model. Finally, the experimental applications and results with a piezotube actuator are detailed in section-5.

\section{MODELING AND IDENTIFICATION OF MONOVARIABLE GENERALIZED BOUC-WEN}

\subsection{Remind of the monovariable Bouc-Wen and Wang-Wen modeling}

Hysteresis in structural materials can be modelled by using the set of equations (1) which is relation between force and material deformation [1].

$$
\left\{\begin{array}{l}
f(x, \dot{x}, z)=\alpha k_{0} x+(1-\alpha) k_{0} z \\
\dot{z}=\dot{x}\left[A-|z|^{m} \psi(x, \dot{x}, z)\right]
\end{array}\right.
$$

$f$ is the resisting force, $x$ and $\dot{x}$ represent respectively the deformation and the velocity of deformation, $\alpha$ is the structure stiffness ratio, $k_{0}$ is the initial stiffness, and $z$ is a variable denoting the structure inelastic behaviour.

$\psi$ is the hysteresis shape control function which is defined according to the hysteresis model. For Bouc-Wen and
Wang-Wen models, $\psi$ functions are represented respectively by (2) and (3):

$$
\begin{gathered}
\psi_{B W}=\gamma+\beta \operatorname{sgn}(\dot{x} z) \\
\psi_{W W}=\gamma+\beta \operatorname{sgn}(\dot{x} z)+\phi[\operatorname{sgn}(\dot{x})+\operatorname{sgn}(z)]
\end{gathered}
$$

The shape control functions for both Bouc-Wen and WangWen models depend only on two variables ( $\dot{x}$ and $z$ ). This allows them to provide four different phases for the shape control, but with one additional parameter $\phi$ for WangWen model.

\subsection{Remind of the monovariable Generalized Bouc-Wen}

In [1], authors extend the number of variables on which depend the hysteresis shape and they include also $x$ in addition to $\dot{x}$ and $z$. This permits them to propose a novel shape control function depending on three variables and to control hysteresis with six shape phases instead of four as in Bouc-Wen and Wang-Wen modelling. Such control function is presented in (4) where $\beta_{i}$ are parameters to be identified.

$$
\begin{aligned}
\psi_{G B W}= & \beta_{1} \operatorname{sgn}(\dot{x} z)+\beta_{2} \operatorname{sgn}(x \dot{x}) \\
& +\beta_{3} \operatorname{sgn}(x z)+\beta_{4} \operatorname{sgn}(\dot{x}) \\
& +\beta_{5} \operatorname{sgn}(z)+\beta_{6} \operatorname{sgn}(x)
\end{aligned}
$$

For the case of piezoelectric based actuators, the first equation of (1) relates the driving signal which is the voltage $U$ with the bending displacement $y$ of the actuator. In addition, the parameter $m$ can be assumed to be equal to 1 due to their elastic structure [21]. So, by combining the first equation of the set in (1) with the control function in (4), the generalized Bouc-Wen model is:

$$
\left\{\begin{array}{l}
y=k U-z \\
\dot{z}=\dot{U}[A-|z| \psi(U, \dot{U}, z)]
\end{array}\right.
$$

with

$$
\begin{aligned}
\psi(U, \dot{U}, z)= & \beta_{1} \operatorname{sgn}(\dot{U} z)+\beta_{2} \operatorname{sgn}(U \dot{U}) \\
& +\beta_{3} \operatorname{sgn}(U z)+\beta_{4} \operatorname{sgn}(\dot{U}) \\
& +\beta_{5} \operatorname{sgn}(z)+\beta_{6} \operatorname{sgn}(U)
\end{aligned}
$$

\section{A NEW MULTIVARIABLE GENERALIZED BOUC-WEN: MODELING AND IDENTIFICATION}

\subsection{Multivariable Generalized Bouc-Wen modeling}

In this section, we propose to extend the monovariable model in equation (5) into a multivariable hysteresis model with $n$ inputs and $n$ outputs.

Let the following expression be the extension of the monovariable model represented in $(5)$ :

$$
\underbrace{\left(\begin{array}{c}
y_{1} \\
y_{2} \\
\vdots \\
y_{n}
\end{array}\right)}_{y}=\underbrace{\left(\begin{array}{cccc}
k_{11} & k_{12} & \cdots & k_{1 n} \\
k_{21} & k_{22} & \cdots & k_{2 n} \\
\vdots & \vdots & & \vdots \\
k_{n 1} & k_{n 2} & \cdots & k_{n n}
\end{array}\right)}_{K} \underbrace{\left(\begin{array}{c}
U_{1} \\
U_{2} \\
\vdots \\
U_{n}
\end{array}\right)}_{U}-\underbrace{\left(\begin{array}{c}
z_{1} \\
z_{2} \\
\vdots \\
z_{n}
\end{array}\right)}_{z}
$$




$$
\begin{gathered}
\underbrace{\left(\begin{array}{c}
\dot{z}_{1} \\
\dot{z}_{2} \\
\vdots \\
\dot{z}_{n}
\end{array}\right)}_{\dot{z}}=\underbrace{\left(\begin{array}{cccc}
A_{11} & A_{12} & \cdots & A_{1 n} \\
A_{21} & A_{22} & \cdots & A_{2 n} \\
\vdots & \vdots & & \vdots \\
A_{n 1} & A_{n 2} & \cdots & A_{n n}
\end{array}\right)}_{A} \underbrace{\left(\begin{array}{c}
\dot{U}_{1} \\
\dot{U}_{2} \\
\vdots \\
\vdots \\
\dot{U}_{n}
\end{array}\right)}_{\dot{A}} \\
-\underbrace{\left(\begin{array}{cccc}
\psi_{11} & \psi_{12} & \cdots & \psi_{1 n} \\
\psi_{21} & \psi_{22} & \cdots & \psi_{2 n} \\
\vdots & \vdots & & \vdots \\
\psi_{n 1} & \psi_{n 2} & \cdots & \psi_{n n}
\end{array}\right)}_{\Psi(U, \dot{U}, z)} \underbrace{\left(\begin{array}{cccc}
\dot{U}_{1} & & & 0 \\
& \dot{U}_{2} & \\
& & \ddots & \\
0 & & \dot{U}_{n}
\end{array}\right)}_{\operatorname{diag}(\dot{U})} \underbrace{\left(\begin{array}{c}
\left|z_{1}\right| \\
\left|z_{2}\right| \\
\vdots \\
\left|z_{n}\right|
\end{array}\right)}_{|z|}
\end{gathered}
$$

i.e.

$$
\left\{\begin{array}{l}
y=K U-z \\
\dot{z}=A \dot{U}-\Psi \operatorname{diag}(\dot{U})|z|
\end{array}\right.
$$

where $|z| \in \mathbb{R}^{n}$ is a state vector and $A \in \mathbb{R}^{n \times n}$, the hysteresis scale control matrix. $\Psi$ is represented by equation (10) and corresponds to the multivariable hysteresis shape control matrix, extended from equation (4).

$$
\begin{aligned}
\Psi(U, \dot{U}, z)= & B \operatorname{diag}(\operatorname{sgn}(\dot{U} z))+C \operatorname{diag}(\operatorname{sgn}(U \dot{U})) \\
+ & D \operatorname{diag}(\operatorname{sgn}(U z))+E \operatorname{diag}(\operatorname{sgn}(\dot{U})) \\
& +F \operatorname{diag}(\operatorname{sgn}(z))+G \operatorname{diag}(\operatorname{sgn}(U))
\end{aligned}
$$

$B, C, D, E, F$ and $G$ are $\mathbb{R}^{n \times n}$ matrices controlling the shape of the multivariable hysteresis.

\subsection{Identification of Multivariable Generalized Bouc-Wen}

By developping equations (7) and (8), (11) can be derived:

$$
\left\{\begin{array}{l}
y_{i}=\left(\sum_{j=1}^{n} k_{i j} U_{j}\right)-z_{i} \\
\dot{z}_{i}=\sum_{j=1}^{n} A_{i j} \dot{U}_{j}-\sum_{j=1}^{n} \psi_{i j} \dot{U}_{j}\left|z_{j}\right| \\
\psi_{i j}=B_{i j} \operatorname{sgn}\left(\dot{U}_{j} z_{j}\right)+C_{i j} \operatorname{sgn}\left(U_{j} \dot{U}_{j}\right)+D_{i j} \operatorname{sgn}\left(U_{j} z_{j}\right) \\
+E_{i j} \operatorname{sgn}\left(\dot{U}_{j}\right)+F_{i j} \operatorname{sgn}\left(z_{j}\right)+G_{i j} \operatorname{sgn}\left(U_{j}\right)
\end{array}\right.
$$

In the above equation, the parameters to be identified are $k_{i j}, A_{i j}, B_{i j} \cdots G_{i j}$ with $1 \leq i \leq n$ and $1 \leq j \leq n$ The subscript $i$ denotes the considered output inside the vector $y$, while $j$ the considered input inside the vector $U$. Furthermore, for $i=j$, we have the direct hysteresis and for $i \neq j$, we have the coupling hysteresis.

To identify the parameters of the new multivariable Generalized Bouc-Wen model hysteresis, the following identification procedure is proposed:

Step 1 (experimental characterization): Apply a periodic (sinusoidal or triangular) input voltage $U_{j}$ (starting by $j=1$ ) to the system and leave all the remaining inputs (i.e. all $U_{k}$ with $k \neq j$ and $1 \leq k \leq n$ ) equal to zero. Capture the output displacement $y_{j}$ due to this voltage $U_{j}$ (direct axis), and capture also the coupling caused by this voltage to the other axis $\left(y_{k}, k \neq j\right)$. In the model, these will correspond to $\left(U_{j}, y_{i}\right)$ (with $i=1, \ldots, j, \ldots n$ ) which, from equation (11), are derived as follows:

$$
\left\{\begin{array}{l}
y_{i}=k_{i j} U_{j}-z_{i} \\
\dot{z}_{i}=A_{i j} \dot{U}_{j}-\psi_{i j} \dot{U}_{j}\left|z_{j}\right| \\
\psi_{i j}=B_{i j} \operatorname{sgn}\left(\dot{U}_{j} z_{j}\right)+C_{i j} \operatorname{sgn}\left(U_{j} \dot{U}_{j}\right)+D_{i j} \operatorname{sgn}\left(U_{j} z_{j}\right) \\
+E_{i j} \operatorname{sgn}\left(\dot{U}_{j}\right)+F_{i j} \operatorname{sgn}\left(z_{j}\right)+G_{i j} \operatorname{sgn}\left(U_{j}\right)
\end{array}\right.
$$

Step 2 (identification of the direct hysteresis parameters): Identify, first, the parameters $k_{j j}, A_{j j} \cdots G_{j j}$ from the direct hysteresis $\left(U_{j}, y_{i}\right)$ (with $i=j$ ). This is done by using the following equations:

$$
\left\{\begin{array}{l}
y_{j}=k_{j j} U_{j}-z_{j} \\
\dot{z}_{j}=A_{j j} \dot{U}_{j}-\psi_{j j} \dot{U}_{j}\left|z_{j}\right| \\
\psi_{j j}=B_{j j} \operatorname{sgn}\left(\dot{U}_{j} z_{j}\right)+C_{j j} \operatorname{sgn}\left(U_{j} \dot{U}_{j}\right)+D_{j j} \operatorname{sgn}\left(U_{j} z_{j}\right) \\
+E_{j j} \operatorname{sgn}\left(\dot{U}_{j}\right)+F_{j j} \operatorname{sgn}\left(z_{j}\right)+G_{j j} \operatorname{sgn}\left(U_{j}\right)
\end{array}\right.
$$

Step 3 (identification of the coupling hysteresis parameters): Then, identify the parameters $k_{j i}, A_{j i} \ldots$ $G_{j i}$ from the coupling hysteresis $\left(\left(U_{j}, y_{i}\right)\right.$ with $\left.i \neq j\right)$. This is done using the following equations:

$$
\left\{\begin{array}{l}
y_{i}=k_{i j} U_{j}-z_{i} \\
\dot{z}_{i}=A_{i j} \dot{U}_{j}-\psi_{i j} \dot{U}_{j}\left|z_{j}\right| \\
\psi_{i j}=B_{i j} \operatorname{sgn}\left(\dot{U}_{j} z_{j}\right)+C_{i j} \operatorname{sgn}\left(U_{j} \dot{U}_{j}\right)+D_{i j} \operatorname{sgn}\left(U_{j} z_{j}\right) \\
+E_{i j} \operatorname{sgn}\left(\dot{U}_{j}\right)+F_{i j} \operatorname{sgn}\left(z_{j}\right)+G_{i j} \operatorname{sgn}\left(U_{j}\right) \\
\dot{z}_{j}=A_{j j} \dot{U}_{j}-\psi_{j j} \dot{U}_{j}\left|z_{j}\right|
\end{array}\right.
$$

After that, repeat the previous three steps for $j=2,3, \ldots, n$ to find the remaining parameters.

Parameters identification at the individual steps Step 2 and Step 3 can be performed with classical technique of identification. This individual identification is similar to the monovariable hysteresis model identification. For instance, in [3], the monovariable Generalized Bouc-Wen parameters were identified by using techniques such as Genetic Algorithms. In this paper, we have performed the identification in the individual steps (Step 2 and Step 3) by using the non-linear least square Matlab optimization toolbox (lsqnonlin) mentioned in [1] to minimize the sum of squared errors over the concerned entire hysteresis loop.

\section{FEEDFORWARD CONTROL OF THE MULTIVARIABLE GENERALIZED BOUC-WEN}

Assuming now that the parameters of the multivariable Bouc-Wen hysteresis model in (9) are identified, we present in this section a (multivariable) compensator for the hysteresis. The compensator is built in a way such that the general feedforward controlled system as depicted in Fig.1a satisfies the following condition:

$$
y^{\text {ref }}=y
$$

where $y^{\text {ref }}\left(\right.$ with $\left.y^{\text {ref }}=\left(y_{1}^{\text {ref }} y_{2}^{\text {ref }} \cdots y_{n}^{\text {ref }}\right)^{T}\right)$ is the desired (reference) input vector. The first equation of the multivariable model in (9) becomes therefore:

$$
y^{r e f}=K U-z
$$

From (16), the compensator is directly yielded as follows which has an inverse multiplicative structure:

$$
U=K^{-1}\left(y^{r e f}+z\right)
$$


where the control voltage $U$ is the output of the compensator and the desired displacement $y^{r e f}$ is its input. It is worth to notice that this compensator is implementable because $K$ is invertible (non singular). Indeed, when designing a multi-DOF actuator, the first natural expectation is that each input control $U_{i}$ affects a corresponding output displacement and axis $y_{i}$ : in other words, the direct transfers $K_{i i}$ are non-null. The only case where $K$ is singular is when applying a voltage $U_{i}$, couplings $y_{j}$ $(j \neq i)$ exist in a such a way that their sum compensates for the the output $y_{i}$. This case is very rare and in general, designers of actuators will never allow systems having such characteristics.

In details, the compensator of equation (17) is written as in (18) while the simplified diagram for the multivariable hysteresis compensator with the proposed approach is represented in Fig.1-b.

$$
\underbrace{\left(\begin{array}{c}
U_{1} \\
U_{2} \\
\vdots \\
U_{n}
\end{array}\right)}_{U}=\underbrace{\left(\begin{array}{cccc}
K_{11} & K_{12} & \cdots & K_{1 n} \\
K_{21} & K_{22} & \cdots & K_{2 n} \\
\vdots & \vdots & & \vdots \\
K_{n 1} & K_{n 2} & \cdots & K_{n n}
\end{array}\right)^{-1}}_{K^{-1}} \underbrace{\left(\begin{array}{c}
y_{1}^{r e f}+z_{1} \\
y_{2}^{r e f}+z_{2} \\
\vdots \\
y_{n}^{r e f}+z_{n}
\end{array}\right)}_{y^{r e f}+z}
$$

The proposed approach is devoted to multi-DOF piezo-

(a)
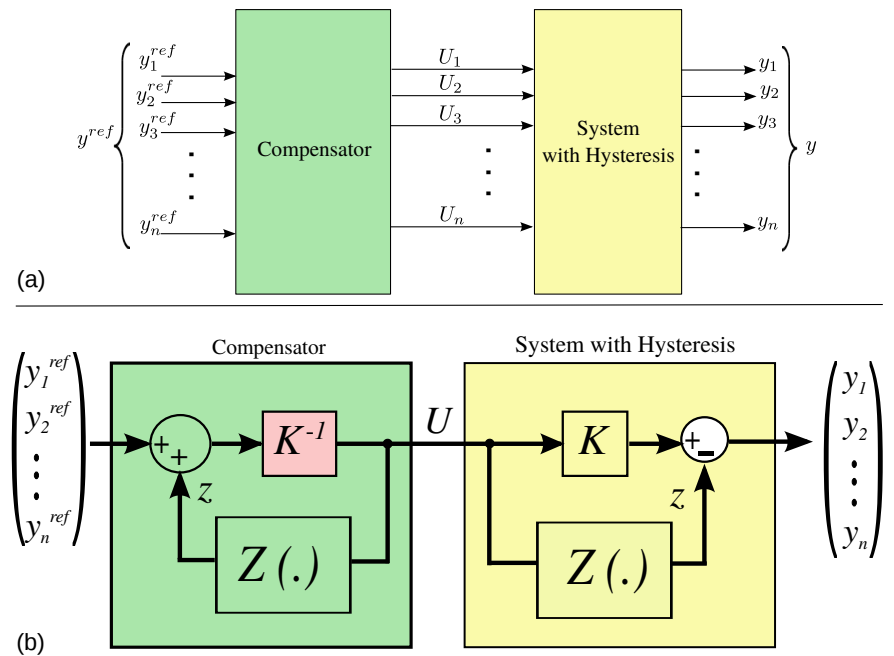

Fig. 1. (a): block diagram of a feedforward control of multivariable hysteresis. (b): Generalized Bouc-Wen based multivariable hysteresis compensator. $Z($.$) de-$ notes a non-linear operator characterizing the second equation of (9)

electric actuators. Another advantage is that there is no additional calculation to derive the compensator, except the inversion of $K$. As soon as the model is identified, the compensator is derived thanks to the inverse multiplicative structure.

\section{EXPERIMENTAL RESULTS AND DISCUSSIONS}

\subsection{Experimental setup}

The actuator used for the experiments is a PT230.94 piezotube scanner (from PIceramic company) often used in atomic force microscopy (AFM). This is an actuator designed to provide deflections along with three directions (X, Y and Z). The actuator is made up of PZT material (lead zirconate titanate), four external electrodes $(+\mathrm{x},-\mathrm{x}$, $+\mathrm{y}$ and $-\mathrm{y})$, and an inside electrode for ground. $+U$ and $-U$ voltages can be applied on $+\mathrm{x}$ and $-\mathrm{x}$ electrodes $(+\mathrm{y}$ and $-\mathrm{y}$ respectively) to obtain tube deflections along the $\mathrm{X}$ direction ( $Y$ direction). Extension (elongation) along the $\mathrm{Z}$ direction is obtained by applying simultaneously $+U$ on the four external electrodes.

The tube deflections are measured by using LC2420 optical displacement sensors (from Keyence Company). These sensors are set to have a resolution of $50 \mathrm{~nm}$, an accuracy of $200 \mathrm{~nm}$ and a bandwidth of $1500 \mathrm{kHz}$.

As PT230.94 operating voltage range is $\pm 250 \mathrm{~V}$, a high voltage amplifier is used to amplify the control voltage. The sensors and the amplifier are connected to the computer through a 1103-dSPACE board (Fig.2).

The tubular shape of the actuator does not allow linear measurement with the optical displacement sensors. For this reason, a small cube (with faces pointing towards the sensors) is placed on the top of the piezotube.

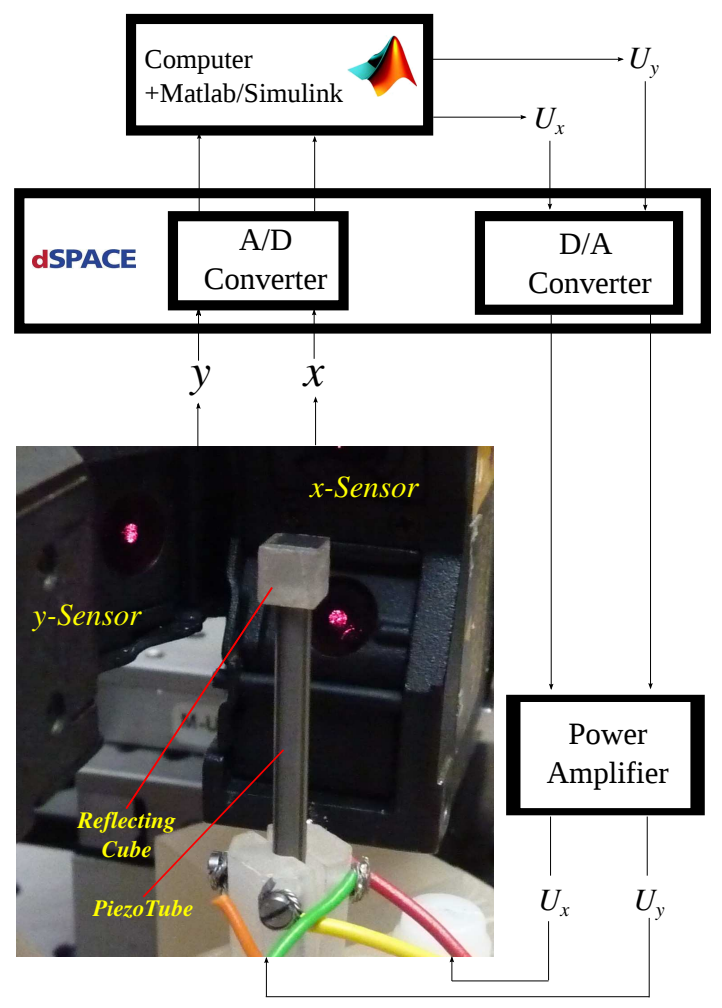

Fig. 2. Experimental setup description.

\subsection{System characterization, modeling and identification}

This section is devoted to the characterization and modeling of the piezotube and the parameters identification.

- Multivariable Hysteresis Characterization:

We apply first a sine voltage $U_{x}$ with an amplitude of $200 \mathrm{~V}$ and a frequency of $0.1 \mathrm{~Hz}$ to the $\mathrm{x}$ electrodes $(+\mathrm{x}$ and $-\mathrm{x})$ and we set $U_{y}$ equal to zero. The resulting displacements $X$ and $Y$ are measured thanks to the optical sensors. Then we plot $X$ versus $U_{x}$ (direct 
transfer) and $Y$ versus $U_{x}$ (coupling). They are pictured in Fig.3a-(1) and in Fig.3a-(3) respectively.

Then, $U_{x}$ is set equal to zero and we apply the same sine voltage but to the y electrodes $(+\mathrm{y}$ and $-\mathrm{y})$. The resulting displacements $X$ and $Y$ are once again measured and we afterwards plot $X$ versus $U_{y}$ (coupling) and $Y$ versus $U_{y}$ (direct transfer). The plots are pictured in Fig.3a-(2) and in Fig.3a-(4) respectively. Notice that the chosen frequency $(0.1 \mathrm{~Hz})$ used for the characterization and identification corresponds to the operational frequency when controlling the system.

- Generalized Bouc-Wen parameters Identification and Validation:

This is done by following the steps discussed in section III.B. From the experimental hysteresis curves, we have identified the parameters of the multivariable Generalized Bouc-Wen model that fits with the experimental data by following these steps and by applying a nonlinear least square optimization with Matlab.

With the identified parameters, the Generalized Bouc-Wen set of equations (7), (8) and (10) can now be written as:

$$
\begin{aligned}
& \left(\begin{array}{l}
X \\
Y
\end{array}\right)=\underbrace{\left(\begin{array}{cc}
0.1873 & 0.0432 \\
-0.0193 & 0.2047
\end{array}\right)}_{K}\left(\begin{array}{l}
U_{x} \\
U_{y}
\end{array}\right)-\left(\begin{array}{l}
z_{x} \\
z_{y}
\end{array}\right) \\
& \left(\begin{array}{l}
\dot{z}_{x} \\
\dot{z_{y}}
\end{array}\right)=\underbrace{\left(\begin{array}{cc}
0.0394 & 0.0336 \\
-0.0195 & 0.0640
\end{array}\right)}_{A}\left(\begin{array}{l}
\dot{U}_{x} \\
\dot{U}_{y}
\end{array}\right) \\
& -\underbrace{\left(\begin{array}{cc}
\psi_{x x} & \psi_{x y} \\
\psi_{y x} & \psi_{y y}
\end{array}\right)}_{\Psi(U, \dot{U}, z)}\left(\begin{array}{cc}
\dot{U}_{x} & 0 \\
0 & \dot{U}_{y}
\end{array}\right)\left(\begin{array}{l}
\left|z_{x}\right| \\
\left|z_{y}\right|
\end{array}\right)
\end{aligned}
$$

with $\Psi(U, \dot{U}, z)=$

$$
\begin{aligned}
\underbrace{\left(\begin{array}{cc}
0.0037 & -0.0006 \\
-0.0015 & 0.0032
\end{array}\right)}_{B}\left(\begin{array}{cc}
\operatorname{sgn}\left(\dot{U}_{x} z_{x}\right) & 0 \\
0 & \operatorname{sgn}\left(\dot{U}_{y} z_{y}\right)
\end{array}\right) \\
+\underbrace{\left(\begin{array}{ll}
0.0224 & 0.0010 \\
0.0021 & 0.0035
\end{array}\right)}_{C}\left(\begin{array}{cc}
\operatorname{sgn}\left(U_{x} \dot{U}_{x}\right) & 0 \\
0 & \operatorname{sgn}\left(U_{y} \dot{U}_{y}\right)
\end{array}\right) \\
+\underbrace{\left(\begin{array}{ll}
-0.0175-0.0004 \\
-0.0002-0.0010
\end{array}\right)}_{D}\left(\begin{array}{cc}
\operatorname{sgn}\left(U_{x} z_{x}\right) & 0 \\
0 & \operatorname{sgn}\left(U_{y} z_{y}\right)
\end{array}\right) \\
+\underbrace{\left(\begin{array}{cc}
0.0013-0.0002 \\
0.0001-0.0003
\end{array}\right)}_{E}\left(\begin{array}{cc}
\operatorname{sgn}\left(\dot{U}_{x}\right) & 0 \\
0 & \operatorname{sgn}\left(\dot{U}_{y}\right)
\end{array}\right) \\
+\underbrace{\left(\begin{array}{ll}
-0.0013-0.0004 \\
-0.0001-0.0002
\end{array}\right)}_{F}\left(\begin{array}{cc}
\operatorname{sgn}\left(z_{x}\right) & 0 \\
0 & \operatorname{sgn}\left(z_{y}\right)
\end{array}\right) \\
+\underbrace{\left(\begin{array}{cc}
0.0003 & 0.0013 \\
-0.0001 & 0.0004
\end{array}\right)}_{F}\left(\begin{array}{rr}
\operatorname{sgn}\left(U_{x}\right) & 0 \\
0 & \operatorname{sgn}\left(U_{y}\right)
\end{array}\right)
\end{aligned}
$$

We mention that the model parameters are identified only from the external hysteresis loop, i.e from the hysteresis loops captured when the input voltage amplitude is in
Table 1. Comparison between system responses, before and after compensation

\begin{tabular}{ccc}
\hline \hline & $\begin{array}{c}\text { Before } \\
\text { Compensation }\end{array}$ & $\begin{array}{c}\text { After } \\
\text { Compensation }\end{array}$ \\
\hline Hysteresis & & \\
$(h / H)$ & & \\
\hline Expected $X$ & $12.8 / 68=18.8 \%$ & $1.8 / 60=3.0 \%$ \\
Expected $Y$ & $11.5 / 63=18.2 \%$ & $0.9 / 60=1.5 \%$ \\
Coupling $X$ & $1 / 3.2=31.2 \%$ & $0.0 / 1.2=0 \%$ \\
Coupling $Y$ & $0.8 / 2.3=34.7 \%$ & $0.0 / 1.1=0 \%$ \\
\hline \hline Couplings & & \\
amplitudes & & $1.1 \mu m$ \\
Coupling $X$ & $3.2 \mu m$ & $1.2 \mu m$ \\
Coupling $Y$ & $2.3 \mu m$ & $1.1 / 60=1.8 \%$ \\
Coupl $X / \operatorname{Exp} X$ & $3.2 / 68=4.7 \%$ & $1.2 / 60=2.0 \%$ \\
Coupl $Y / \operatorname{Exp} Y$ & $2.3 / 63=3.6 \%$ & \\
\hline \hline
\end{tabular}

its maximal operating range $(200 \mathrm{~V})$. Fig.3b-(1), (2), (3) and (4) picture the comparison between the experimental hysteresis and the simulation of the identified model. In the figures, hysteresis with the external loop and hysteresis obtained with lower voltages are plotted. This comparison shows the good conveniency of the identified model. The efficiency of the Generalized Bouc-Wen modeling is very remarkable in Fig.3b-(2) and (3) where the asymmetry of the hysteresis is important. This asymmetry cannot be tracked by the classical Bouc-Wen model.

\subsection{Hysteresis compensation and Comparison between compensated and uncompensated systems}

After the model identification and validation, we have implemented in the Matlab-Simulink software the hysteresis compensator of Fig.1-b. The compensator is built by using the parameters identified in section V-B. When the piezotube actuator is controlled, the output deflections $X$ and $Y$ versus the input references $X_{\text {ref }}$ and $Y_{\text {ref }}$ are represented in Fig.4.

Comparing the initial hysteresis in Fig.3a with the results in Fig.4 obtained with the compensator, we can clearly see that the proposed compensator has considerably suppressed the hysteresis along $\mathrm{X}$ and along $\mathrm{Y}$ axis, and reduced the coupling amplitudes. Table.1 summarizes the performances of the piezotube with and without the proposed hysteresis compensator. The hysteresis which were initially $18.8 \%$ and $18.2 \%$ for the $X$ and $Y$ displacements respectively were reduced to less than $3 \%$ and than $1.5 \%$ (direct transfers). We also remark that the input-output map of these direct transfers is with unitary gain. Concerning the couplings hysteresis, they were reduced from $31.2 \%$ and $34.7 \%$ for the $X$ and $Y$ displacements respectively to almost $0 \%$. The amplitudes of the couplings were also reduced. The remaining couplings are due to the fact that the coupling hysteresis of the piezotube actuator were not regular and then the generalized Bouc-Wen technique could not exactly be modelled. This non-regularity involves other kinds of nonlinearities to be combined with the hysteresis, which is not the objective of this paper.

\subsection{Lissajous curves trajectories tracking}

Lissajous trajectories are used in [5] as a novel reference inputs for high speed scanning probe microscopy. In this 

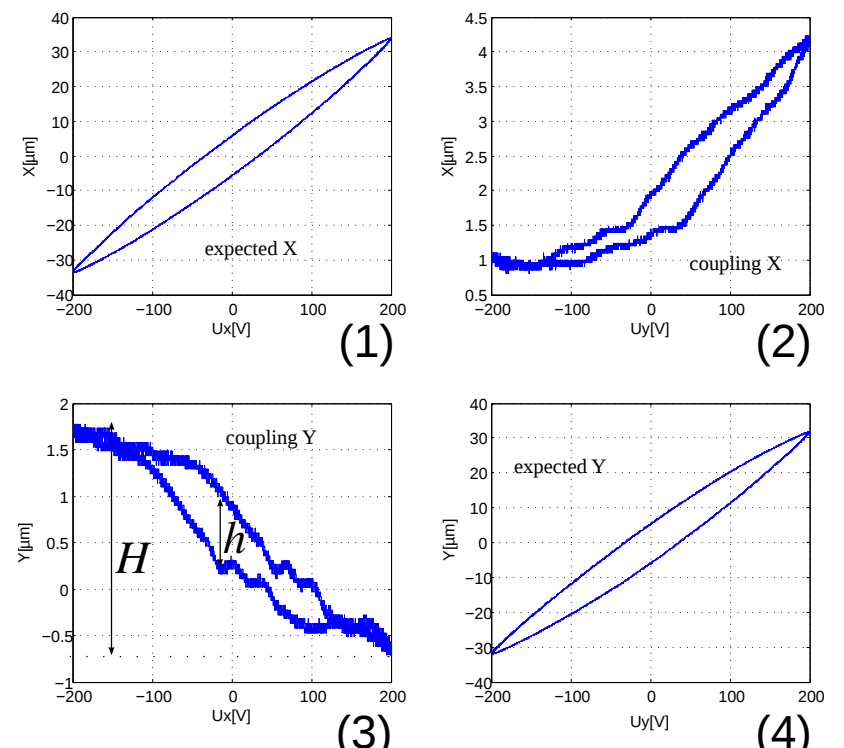

(4)

(a) Hysteresis Characterization: (1) and (3), $X$ and $Y$ displacements when only $U_{x}$ is applied $\left(U_{y}=0\right)$, and (2) and (4) when only $U_{y}$ is applied $\left(U_{x}=0\right)$.
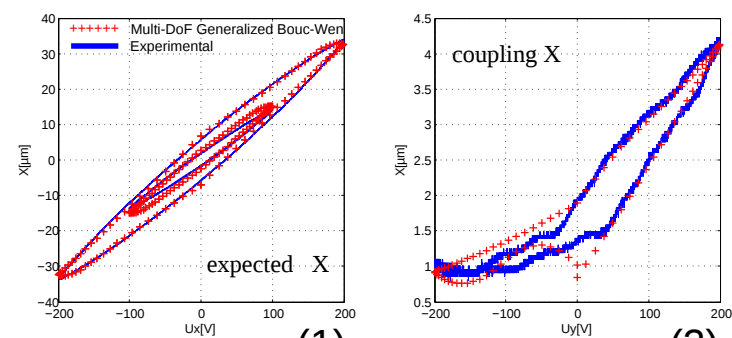

(1)

(2)

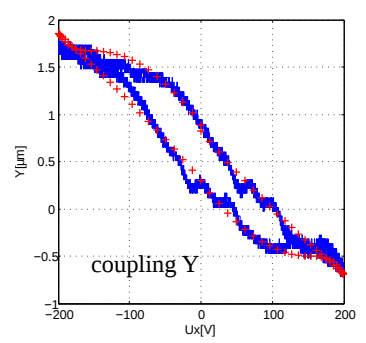

(3)

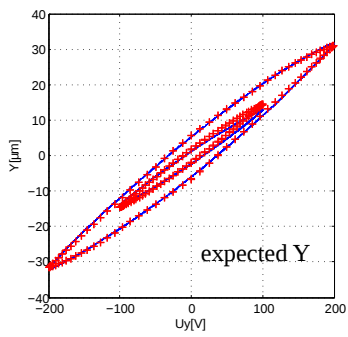

(4)

(b) Experimental curves and the simulation of the identified 2-DoF Generalized Bouc-Wen model with different hysteresis loops.

Fig. 3. Hysteresis characterization and Multivariable Generalized Bouc-Wen model representation.

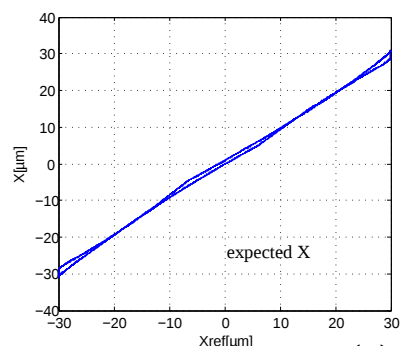

(a)

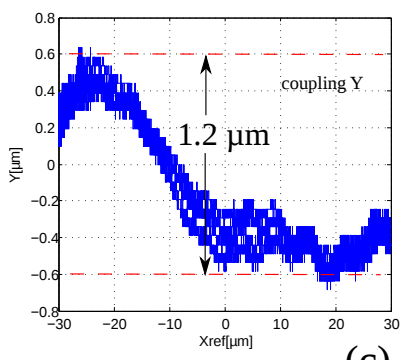

(c)

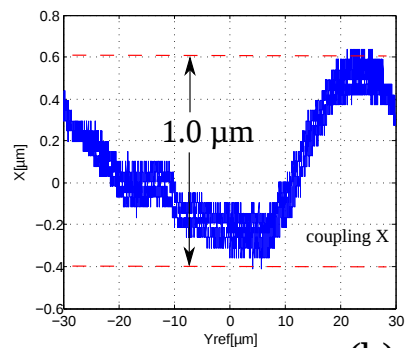

(b)

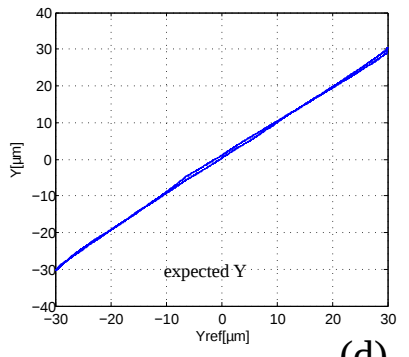

(d)

Fig. 4. Deflections of the piezotube actuator versus the reference inputs when using the compensator. (a) and (d) show the expected deflections, and (b) and (c), the couplings which are reduced.

subsection, we test the efficiency of our compensator by executing Lissajous trajectory tracking in X-Y plane. The tracked figure is based on two input sinusoidal signals: $X_{\text {ref }}=\hat{X} \sin \left(2 \pi f_{x} t+\phi\right)$ and $Y_{\text {ref }}=\hat{Y} \sin \left(2 \pi f_{y} t\right)$. The experimental results which are reported in Fig.5 were obtained at a frequency ratio $f_{x} / f_{y}=0.3 \mathrm{~Hz} / 0.2 \mathrm{~Hz}=3 / 2$ and $\phi=\pi / 2$. When using the compensator, the references have signal amplitudes $\hat{X}$ and $\hat{Y}$ equal to $30 \mu m$. For the uncompensated system, the applied input voltages are $U_{x}=200 \sin \left(2 \pi f_{x} t+\phi\right)$ and $U_{y}=200 \sin \left(2 \pi f_{y} t\right)$. This amplitude voltage $(200 \mathrm{~V})$ corresponds to about $30 \mu \mathrm{m}$ of

deflections according to Fig.3a. The results were afterwards scaled in order to make the comparison coherent. From this figure, we notice the improvement of contour tracking when using the proposed Generalized Bouc-Wen hysteresis compensator.
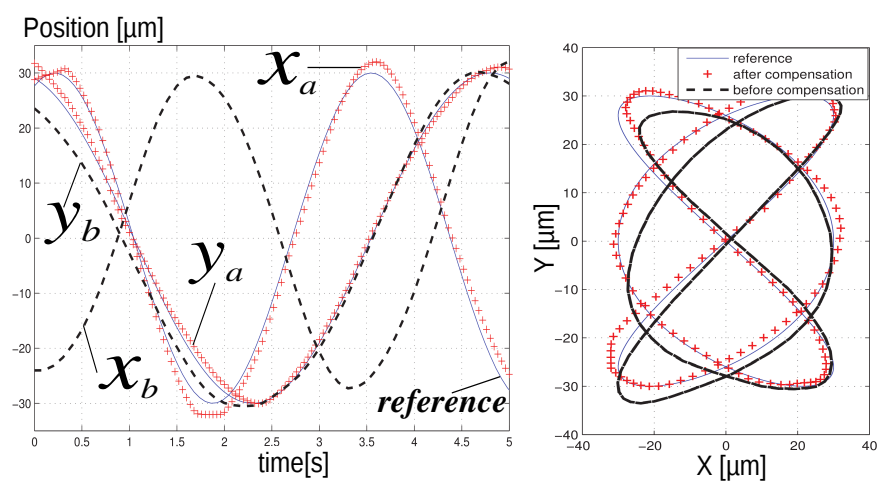

Fig. 5. Lissajous curves tracking before and after compensation. Subscripts $a$ and $b$ stand respectively for $a f t e r$ and before compensation

\section{CONCLUSION AND PERSPECTIVES}

A multivariable generalized Bouc-Wen model for multiDoF systems with hysteresis has been presented in this paper. The proposed model is applicable to multi-DoF piezoelectric actuators exhibiting asymmetric hysteresis both in the expected (direct) transfers and in the couplings transfers. Then, a procedure to identify the parameters of the multivariable model has been proposed. Afterwards, we have proposed a compensator devoted to the multivariable generalized Bouc-Wen model. Based on the inverse multiplicative structure, there is no additional calculation of the compensator. The ease of implementation of the compensator was also discussed. Finally, the proposed 
modeling, identification procedure and compensation technique have been applied to a piezoelectric tube scanner often used in atomic force microscopy and working in 2-DoF. Experimental tests with classical signal references and more complex references trajectories (Lissajous curves) demonstrated the efficiency of the proposed approach.

Future work will be focused on the adaptation/modification of the proposed approach in order to take into account the modeling and compensation of multivariable hysteresis with rate-dependent feature.

\section{REFERENCES}

[1] Song, Junho and Der Kiureghian, Armen, "Generalized Bouc-Wen model for highly asymmetric hysteresis", Journal of engineering mechanics, pp. 610-618, 2006

[2] Jiang, Hao and Ji, Hongli and Qiu, Jinhao and Chen, Yuansheng, "A modified prandtl-ishlinskii model for modeling asymmetric hysteresis of piezoelectric actuators, Ultrasonics, Ferroelectrics and Frequency Control", IEEE Transactions on, pp. 1200-1210, 2010

[3] Tudor Sireteanu, Marius Giuclea, Ana Maria Mitu and Gheorghe Ghita, "A Genetic Algorithms Method for Fitting the Generalized Bouc-Wen Model to Experimental Asymmetric Hysteretic Loops", ASME DC Journal of Vibration and Acoustics, pp. 134-144 vol.4, 2012

[4] Ismail, Mohammed and Ikhouane, Fayçal and Rodellar, José, "The hysteresis Bouc-Wen model, a survey", Springer, Archives of Computational Methods in Engineering, pp.161-188, 2009

[5] Tuma, Tomas and Lygeros, John and Sebastian, Abu and Pantazi, Angeliki, "Optimal scan trajectories for high-speed scanning probe microscopy", American Control Conference (ACC), pp.3791-3796, 2012

[6] Rakotondrabe, Micky, "Bouc-Wen modeling and inverse multiplicative structure to compensate hysteresis nonlinearity in piezoelectric actuators", IEEE Transactions on Automation Science and Engineering, pp.428-431 Vol.8, 2011

[7] Jang, Ming-Jyi and Chen, Chieh-Li and Lee, JieRen,"Modeling and control of a piezoelectric actuator driven system with asymmetric hysteresis", Journal of the Franklin Institute, Elsevier, pp.17-32, 2009

[8] Devasia, Santosh and Eleftheriou, Evangelos and Moheimani, SO Reza," A survey of Control Issues in Nanopositioning", IEEE Transactions on Control Systems Technology, pp.802-823 Vol.15, 2007

[9] Merry, Roel and Uyanik, Mustafa and Van De Molengraft, René and Steinbuch, Maarten and Koops, Richard and Van Veghel, Marijn, "Modeling and compensation of asymmetric hysteresis in a piezo actuated metrological AFM", American Control Conference $A C C^{\prime}$ 09, pp. 967-972, 2009

[10] Clayton, GM and Tien, S and Fleming, AJ and Moheimani, SOR and Devasia, S, "Hysteresis and vibration compensation in piezoelectric actuators by integrating charge control and inverse feedforward", 4th IFAC Symp. Mechatron. Syst., Heidelberg, Germany, 2006

[11] Kuhnen, Klaus and Janocha, Hartmut, "Inverse feedforward controller for complex hysteretic nonlinear- ities in smart-material systems", Control Intelligent Systems, pp.74-83 Vol.29, 2001

[12] Banks, HT and Smith, Ralph C, "Hysteresis modeling in smart material systems ", International conference on micromechanics, intelligent materials and robotics, pp.675-682, 1998

[13] R. V. Iyer, X. Tan, P. S. Krishnaprasad, "Approximate inversion of the Preisach hysteresis operator with application to control of smart actuators", IEEE Transactions on Automatic Control, vol. 50, no. 6, pp. 798-810, 2005

[14] Merry, Roel and Uyanik, Mustafa and van de Molengraft, René and Koops, Richard and van Veghel, Marijn and Steinbuch, Maarten, "Identification, control and hysteresis compensation of a 3 DOF metrological AFM", Asian Journal of Control, pp.130-143 Vol.11, 2009

[15] Yong, Yuen Kuan and Aphale, Sumeet S and Moheimani, SO Reza, "Design, identification, and control of a flexure-based XY stage for fast nanoscale positioning", IEEE Transactions on Nanotechnology, pp.46 Vol.8, 2009

[16] Rakotondrabe, Micky and Agnus, Joël and Lutz, Philippe, "Feedforward and IMC-feedback control of a nonlinear 2-DOF piezoactuator dedicated to automated tasks", IEEE Conference on Automation Science and Engineering, pp.393-398, 2011

[17] Micky Rakotondrabe and Kanty Rabenorosoa and Joël Agnus and Nicolas Chaillet, "Robust Feedforward-Feedback Control of a Nonlinear and Oscillating 2-DOF Piezocantilever", IEEE T. Automation Science and Engineering, pp.506-519 Vol.8, 2011

[18] Xu, Qingsong and Li, Yangmin, "Dahl model-based hysteresis compensation and precise positioning control of an XY parallel micromanipulator with piezoelectric actuation", Journal of dynamic systems, measurement, and control, pp.041011 Vol.132, 2010

[19] Al Janaideh, Mohammad and Su, Chun-Yi and Rakheja, Subhash, "Compensation of rate-dependent hysteresis nonlinearities in a piezo micro-positioning stage", International Conference on Robotics and Automation (ICRA), pp.512-517, 2010

[20] Ang, Wei Tech and Khosla, Pradeep K and Riviere, Cameron N, "Feedforward controller with inverse rate-dependent model for piezoelectric actuators in trajectory-tracking applications", Transactions on Mechatronics, IEEE/ASME, pp.134-142 Vol.12, 2007

[21] Low, TS and Guo, W, "Modeling of a three-layer piezoelectric bimorph beam with hysteresis", Journal of Microelectromechanical Systems, pp.230-237 Vol.4, 1995

[22] Sain, Patrick M and Sain, Michael K and Spencer, BF, "Models for Hysteresis and Application to Structural Control", American Control Conference, 1997 